Arq. Bras. Med. Vet. Zootec., v.67, n.3, p.846-854, 2015

\title{
Parâmetros genéticos para características de prolificidade nas raças Landrace e Large White
}

\author{
[Genetic parameters for prolificacy in Landrace and Large White breeds] \\ M. Anrain ${ }^{1}$, J.A.G. Bergmann ${ }^{1}$, R. Irgang $^{2}$, B.D. Valente ${ }^{3}$ \\ ${ }^{1}$ Escola de Veterinária - Universidade Federal de Minas Gerais - Belo Horizonte, MG \\ ${ }^{2}$ Centro de Ciências Agrárias - Universidade Federal de Santa Catarina - Florianópolis, SC \\ ${ }^{3}$ University of Wisconsin - Madison
}

\begin{abstract}
RESUMO
Dados de suínos das raças Landrace (LD) e Large White (LW) foram utilizados para estimar componentes de variância para número total de leitões nascidos (NTN), nascidos vivos (NV) e de leitões vivos ao quinto dia (LV5). Usou-se o método da máxima verossimilhança restrita (REML) para estimar componentes de variância. O modelo misto incluiu os efeitos fixos de mês e ano de nascimento e da inseminação da porca e ordem de parto. Análises unicaracterísticas incluíram os efeitos genético direto, genético materno e de ambiente permanente. Análises multicaracterísticas foram feitas para estimar correlações genéticas. Os modelos unicaracterísticas foram comparados e o que continha apenas o efeito genético direto foi considerado o mais adequado. As estimativas de herdabilidade para NTN foram de 0,15 para LW e de 0,08 a 0,12 para LD, dependendo do modelo, para NV foram de 0,14 para LW e de 0,05 a 0,12 para LD, e para LV5, variaram de 0,11 a 0,12 para $\mathrm{LW}$ e de 0,03 a 0,08 para LD. As correlações fenotípicas e genéticas entre as três características foram altas e favoráveis. Conclui-se que a seleção para aumento do LV5 pode ser uma via interessante para o aumento do tamanho da leitegada, da sobrevivência de leitões e da habilidade materna em suínos.
\end{abstract}

Palavras-chave: herdabilidade, prolificidade, resposta à seleção

\begin{abstract}
Data from Landrace $(L D)$ and Large White $(L W)$ sows were analyzed to estimate variance components for total number of piglets born (NTN), number of piglets born alive (NV) and number alive on day 5 after birth (LV5). REML mixed model equations included the fixed effects of sow's month and year of birth and insemination and farrowing order. Univariate analyses included, alternatively, direct genetic, maternal genetic and permanent environmental effects. Multiple trait analysis was performed to estimate genetic correlations among the traits. Comparisons between univariate models indicated that the model containing only direct genetic effect was the most appropriate for parameter estimation. Estimates of heritability for NTN were 0.15 in $L W$ and ranged from 0.08 to .12 in $L D, 0.14$ in LW, from 0.05 to 0.12 in $L D$ for $N V$, from 0.11 to 0.12 in $L W$, and from 0.03 to 0.08 in LD for LV5. Phenotypic and genetic correlations among traits were high and favorable. Results suggest that selection for LV5 is an interesting alternative way to increase litter size, piglet survival and maternal ability in swine.
\end{abstract}

Keywords: heritability, litter size, selection response

\section{INTRODUÇÃO}

A suinocultura apresentou grandes avanços nos últimos anos, graças aos esforços feitos nas áreas de melhoramento genético, nutrição e manejo

Recebido em 4 de março de 2013

Aceito em 16 de março de 2015

E-mail: anrain@gmail.com dos animais. Tais avanços resultaram em grandes transformações em características de carcaça e eficiência reprodutiva na população, o que permitiu o aumento da produtividade em todos os setores da atividade. Como exemplo, houve incremento no número de leitões nascidos por 
leitegada de 3,28 leitões na raça Landrace e de 3,89 na raça Large White, entre 1970 e 2005 (Fávero e Figueiredo, 2009).

O tamanho da leitegada ao desmame é considerado uma das características mais importantes na produção de suínos (SU et al., 2007). Apesar de o número de leitões desmamados por leitegada ser a característica relacionada à prolificidade de maior impacto econômico, a implementação de seleção direta para essa característica é difícil. Uma prática comum nos sistemas de produção de suínos é a uniformização das leitegadas por meio da troca de leitões entre as fêmeas que tiveram parição em momentos próximos. Tal prática resulta em benefícios para o sistema de produção, mas faz com que o tamanho de leitegada à desmama não seja um adequado indicativo da prolificidade da fêmea. Por outro lado, o uso dessa característica para avaliação genética visando à seleção para prolificidade faria sentido se fosse feita com base em fenótipos mensurados na ausência de uniformização das leitegadas. Como tais informações não são disponíveis para ajuste de modelos mistos, estimar adequadamente parâmetros genéticos para o tamanho da leitegada ao desmame é difícil. Os efeitos genéticos para tamanho de leitegada à desmama na presença de uniformização parecem indicar apenas a capacidade de cada fêmea manter a progênie viva a partir da uniformização. Por outro lado, efeitos genéticos obtidos na ausência de uniformização seriam combinações dos méritos para prolificidade e para sobrevivência. É importante ressaltar que acuradas estimativas de parâmetros genéticos para qualquer característica são essenciais para estimação dos valores genéticos e otimização da predição da resposta genética à seleção.

Para superar essa limitação, programas de melhoramento genético de suínos têm realizado seleção para tamanho da leitegada ao nascimento, visando ao efeito indireto no mérito genético para tamanho da leitegada ao desmame. Esse foi, por muito tempo, um importante objetivo de seleção na suinocultura moderna. Porém, o aumento do número de leitões nascidos foi acompanhado por redução na sobrevivência até o desmame (Lund et al., 2002; Bouquet et al., 2006). A mortalidade entre o parto e a desmama foi apontada por Su et al. (2006) como o maior problema na produção de suínos. Um aspecto importante é que cerca de $90 \%$ da mortalidade de leitões na fase pré-desmame acontece entre o primeiro e o quinto dia de vida, de modo que o número de leitões vivos ao quinto dia tem alta correlação $(\mathrm{r}=0,99)$ com o número de leitões desmamados (SU et al., 2007). Portanto, uma alternativa à seleção tradicional para prolificidade é utilzar preditores de efeitos genéticos para número de leitões vivos ao quinto dia. A seleção para essa característica contempla vários componentes de tamanho de leitegada ao desmame, tais como a sobrevivência dos leitões, a habilidade materna e a produção leiteira da fêmea.

O objetivo deste trabalho foi o de estimar parâmetros genéticos e fenotípicos em populações de Landrace e Large White para características de prolificidade em suínos, utilizando modelos estatísticos que incluíram diferentes combinações de efeitos genéticos diretos, genéticos maternos e de ambiente permanente, em análises uni e multicaracterísticas.

\section{MATERIAL E MÉTODOS}

Os dados utilizados foram coletados entre julho de 2005 a janeiro de 2011, em uma unidade de melhoramento genético, localizada no Estado de Minas Gerais. Todas as fêmeas foram inseminadas artificialmente com, pelo menos, duas doses de sêmen. O pedigree disponível para fêmeas e machos continha informações de parentesco que se estendem até não menos do que sete gerações anteriores em relação aos indivíduos com observações.

O banco de dados era composto de animais das raças puras Landrace (LD) e Large White (LW), e continha as seguintes informações: número total de leitões nascidos (NT); número de leitões nascidos vivos (NV); número de leitões vivos ao quinto dia (LV5); data de nascimento da fêmea (mãe da leitegada); data do parto; pedigree completo dos machos e das fêmeas da granja.

Foram considerados na análise estatística os dados da primeira até a sexta parição de cada fêmea. Todos os partos de cada fêmea foram considerados como repetições de uma mesma característica. Foram excluídos dados de NV e LV5 superiores a 20 leitões. Qualquer fêmea que não apresentasse informações sobre data de 
nascimento, data de inseminação ou raça da leitegada foi excluída das análises. Grupos de contemporâneas de ano-mês de nascimento das porcas ou de ano-mês de parição das leitegadas com menos de cinco observações em cada grupo também foram excluídos. Após as restrições, o banco de dados para LW continha observações das três características (NTN, NV e LV5) para 2.366 leitegadas, atribuídas a 862 fêmeas, e aproximadamente de 6.000 animais foram considerados na construção da matriz de parentesco. Para LD, o banco de dados continha informações das mesmas características associadas a 2.432 leitegadas atribuídas a 930 fêmeas, bem como matriz de parentesco de dimensões similares à construída para LW .

As estimativas de componentes de (co)variância (assim como herdabilidades e correlações decorrentes de tais componentes) foram obtidas com base nos modelos mistos unicaracterísticas e multicaracterísticas. As análises dos dados foram realizadas utilizando os quatro modelos apresentados a seguir:

Modelo 1: $\mathrm{y}=\mathrm{X} \beta+\mathrm{Za}+\mathrm{e}$;

Modelo 2: $\mathrm{y}=\mathrm{X} \beta+\mathrm{Za}+\mathrm{Pm}+\mathrm{e}$;

Modelo 3: $\mathrm{y}=\mathrm{X} \beta+\mathrm{Za}+\mathrm{Cp}+\mathrm{e}$;

Modelo 4: $\mathrm{y}=\mathrm{X} \beta+\mathrm{Za}+\mathrm{Pm}+\mathrm{Cp}+\mathrm{e}$

em que: y é o vetor de observações (número total de leitões nascidos ou número de leitões nascidos vivos ou número de leitões vivos ao quinto dia ou todas, nas análises multicaracterísticas); B é o vetor de efeitos fixos (mês e ano do nascimento da fêmea, mês e ano da inseminação artificial da fêmea e ordem de parto.); a é o vetor dos efeitos genéticos aditivos da fêmea; $\mathbf{m}$ é o vetor dos efeitos maternos da mãe da fêmea; $\mathbf{p}$ é o vetor dos efeitos de ambiente permanente; e e é o vetor dos resíduos. $\mathbf{X}, \mathbf{Z}, \mathbf{P}$ e $\mathbf{C}$ são as matrizes de incidência dos efeitos em $\mathbf{B}, \quad \mathbf{a}, \quad \mathbf{m}$ e $\quad \mathbf{p}$, respectivamente.

Assume-se que $\mathrm{E}(\mathbf{y})=\mathbf{X} \boldsymbol{\beta}$, uma vez que os demais efeitos são considerados como aleatórios com esperança igual a zero. A descrição da dispersão esperada para os vetores que contêm esses efeitos para o modelo 4, ou seja, o modelo mais complexo, foi:

$$
V\left[\begin{array}{c}
a \\
m \\
p \\
e
\end{array}\right]=\left[\begin{array}{cccc}
A \sigma_{a}^{2} & A \sigma_{a m} & A \sigma_{a p} & 0 \\
A \sigma_{a m} & A \sigma_{m}^{2} & A \sigma_{m p} & 0 \\
A \sigma_{a p} & A \sigma_{m p} & I \sigma_{p}^{2} & 0 \\
0 & 0 & 0 & I \sigma_{e}^{2}
\end{array}\right]
$$

em que $\sigma_{\mathrm{a}}{ }^{2}$ é a variância genética aditiva direta, $\sigma_{\mathrm{m}}{ }^{2}$ é a variância genética aditiva materna, $\sigma_{\mathrm{p}}{ }^{2}$ é a variância de ambiente permanente da fêmea, $\sigma_{\mathrm{e}}{ }^{2}$ é a variância residual, $\sigma_{\mathrm{am}}$ é a covariância entre os efeitos genético aditivo e materno, $\sigma_{\text {ap }}$ é a covariância entre os efeitos genético aditivo e de ambiente permanente da fêmea, $\sigma_{\mathrm{mp}}$ é a covariância entre os efeitos materno e de ambiente permanente, A é a matriz de parentesco entre todos os animais e I é a matriz identidade.

A variância fenotípica pode ser representada da seguinte maneira, de acordo com cada modelo:

Modelo 1: $\operatorname{Var}(\mathrm{y})=\mathrm{ZAZ}{ }^{\prime} \sigma_{\mathrm{a}}^{2}+\mathrm{I}^{2}{ }_{\mathrm{e}}$;

Modelo 2: $\operatorname{Var}(\mathrm{y})=\mathrm{ZAZ}^{\prime} \sigma_{\mathrm{a}}^{2}+\mathrm{PAP}^{\prime} \sigma_{\mathrm{m}}^{2}+$ $\left(\mathrm{PAZ}+\mathrm{ZAP}{ }^{\prime}\right) \sigma_{\mathrm{am}}+\mathrm{I} \sigma_{\mathrm{e}}^{2}$;

Modelo 3: Var (y) $=$ ZAZ' $\sigma^{2}{ }_{\mathrm{a}}+\mathrm{CC}^{\prime} \sigma_{\mathrm{p}}^{2}+\mathrm{I}^{2}{ }_{\mathrm{e}}$;

Modelo 4: $\operatorname{Var}(\mathrm{y})=\mathrm{ZAZ}{ }^{\prime} \sigma_{\mathrm{a}}^{2}+\mathrm{PAP}^{\prime} \sigma_{\mathrm{m}}^{2}+$ $\left(\mathrm{PAZ}+\mathrm{ZAP} \mathrm{P}^{\prime}\right) \sigma_{\mathrm{am}}^{2}+\mathrm{CC}^{\prime} \sigma_{\mathrm{p}}^{2}+\mathrm{I}_{\mathrm{e}}^{2}$;

Para o modelo 4, as equações em forma matricial são representadas como segue:

$$
\left[\begin{array}{cccc}
X^{\prime} X & X^{\prime} Z_{1} & X^{\prime} Z_{2} & X^{\prime} Z_{3} \\
Z_{1}^{\prime} X & Z_{1}^{\prime} Z_{1}+A^{-1} k_{11} & Z_{1}^{\prime} Z_{2}+A^{-1} k_{12} & Z_{1}^{\prime} Z_{3} \\
Z_{2}^{\prime} X & Z_{2}^{\prime} Z_{1}+A^{-1} k_{21} & Z_{2}^{\prime} Z_{2}+A^{-1} k_{22} & Z_{2}^{\prime} Z_{3} \\
Z_{3}^{\prime} X & Z_{3}^{\prime} Z_{1} & Z_{3}^{\prime} Z_{2} & Z_{3}^{\prime} Z_{3}+I \beta
\end{array}\right] \times\left[\begin{array}{c}
\beta \\
a \\
m \\
l
\end{array}\right]=\left[\begin{array}{c}
X^{\prime} y \\
Z_{1}^{\prime} y \\
Z_{2}^{\prime} y \\
Z_{3}^{\prime} y
\end{array}\right]
$$

Em que:

$$
\left[\begin{array}{lll}
k_{11} & k_{12} & k_{13} \\
k_{21} & k_{22} & k_{23} \\
k_{31} & k_{32} & k_{33}
\end{array}\right]=\left[\begin{array}{ccc}
\sigma_{a}^{2} & \sigma_{a m} & \sigma_{a p} \\
\sigma_{a m} & \sigma_{m}^{2} & \sigma_{m p} \\
\sigma_{a p} & \sigma_{m p} & \sigma_{p}^{2}
\end{array}\right]^{-1} \sigma_{e}^{2} \text { e } \beta=\sigma_{e}^{2} / \sigma_{p}^{2} .
$$


A variância fenotípica é igual a:

$\sigma_{a}^{2}+\sigma_{m}^{2}+\sigma_{a m}+\sigma_{p}^{2}+\sigma_{e}^{2}$

Os componentes de variância e as estimativas de herdabilidade para tamanho de leitegada em diferentes estádios foram estimados para três características (NT, NV, LV5), em análises individuais (unicaracterísticas) e em análises conjuntas das três características (multicaracterísticas).

O banco de dados foi editado e a importância dos efeitos fixos foi avaliada utilizando-se o programa SAS (Statistical..., 2001). Os efeitos fixos foram utilizados na formação dos grupos contemporâneos e incorporados ao modelo para estimar componentes de (co)variância para as características analisadas. Tais estimadores foram obtidos por métodos de Máxima Verossimilhança Restrita, por meio do algoritmo EM (Expectation Maximization), disponível no aplicativo computacional WOMBAT, desenvolvido por Meyer (2007). A cada iteração, o valor do logaritmo da função de máxima verossimilhança foi calculado, sendo adotada como critério de convergência uma diferença entre os valores provenientes de ciclos consecutivos igual ou inferior a $10^{-9}$. Devido a limitações do programa utilizado, apenas informação da primeira leitegada das fêmeas foi utilizada para ajustar os modelos multicaracterísticas. Nesse caso, os efeitos de ambiente permanente foram excluídos do modelo. Para a comparação de modelos, foram utilizados os valores do critério de informação Akaike, ou AIC (Akaike Information Criterion, Akaike 1973), e o critério de informação Bayesiano, ou BIC (Bayesian Information Criterion, Schwarz 1978).

\section{RESULTADOS E DISCUSSÃO}

As estatísticas descritivas para cada raça e característica são apresentadas na Tabela 1. Não houve diferença estatística entre raças para NTN, NV e LV5. A variação, representada pelo desvio padrão das características e pelo coeficiente de variação, foi maior para $\mathrm{LW}$ do que para $\mathrm{LD}$, contrariando os dados obtidos por $\mathrm{Su}$ et al. (2007), que analisaram dados das mesmas características e raças em populações dinamarquesas, e observaram maior variância fenotípica em LD.

Como apresentado na Figura 1, a média observada de LV5 cresceu de 11,36 para 12,39 entre os anos de 2006 e 2010 na raça Landrace, e de 11,57 em 2006 para 12,94 em 2009 na raça Large White.

Os resultados dos componentes de variância para LW e LD são apresentados na Tabela 2.

Com base nas variâncias estimadas, foram calculadas a herdabilidade no sentido restrito, $\left(\mathrm{h}^{2}\right)$, a herdabilidade do efeito materno $\left(\mathrm{h}^{2}{ }_{\text {mater }}\right)$ e a repetibilidade $(\mathrm{R})$ das características para as duas raças, conforme apresentado na Tabela 3.

Tabela 1. Média, desvio padrão e coeficiente de variação para as características de leitegada nas raças Large White e Landrace

\begin{tabular}{|c|c|c|c|c|c|c|c|c|}
\hline \multirow[b]{2}{*}{ Carac. } & \multicolumn{4}{|c|}{ Large White } & \multicolumn{4}{|c|}{ Landrace } \\
\hline & $\begin{array}{c}\mathrm{N}^{\circ} \mathrm{de} \\
\text { leitegada } \\
\mathrm{S}\end{array}$ & Média & $\begin{array}{l}\text { Desvio } \\
\text { padrão }\end{array}$ & $\begin{array}{c}\text { Coef. } \\
\text { variação } \\
\%\end{array}$ & $\begin{array}{c}\mathrm{N}^{\circ} \mathrm{de} \\
\text { leitegadas }\end{array}$ & Média & $\begin{array}{l}\text { Desvio } \\
\text { padrão }\end{array}$ & $\begin{array}{c}\text { Coef. } \\
\text { variação } \\
\%\end{array}$ \\
\hline NTN & 2365 & $14,14 a$ & 3,83 & 27,07 & 2432 & $14,29 \mathrm{a}$ & 3,36 & 23,48 \\
\hline NV & 2365 & $12,44 \mathrm{a}$ & 3,35 & 26,89 & 2432 & $12,55 \mathrm{a}$ & 3,08 & 24,55 \\
\hline LV5 & 2365 & $11,97 \mathrm{a}$ & 3,42 & 28,56 & 2432 & $11,92 \mathrm{a}$ & 3,26 & 27,36 \\
\hline
\end{tabular}

NTN: Número total de leitões nascidos; NV: Número de leitões nascidos vivos; LV5: número de leitões vivos ao quinto dia de vida.

${ }^{\mathrm{a}}$ Médias com a mesma letra na mesma linha indicam não haver diferença significativa entre raças ao nível de $\mathrm{P}<0,05$. 

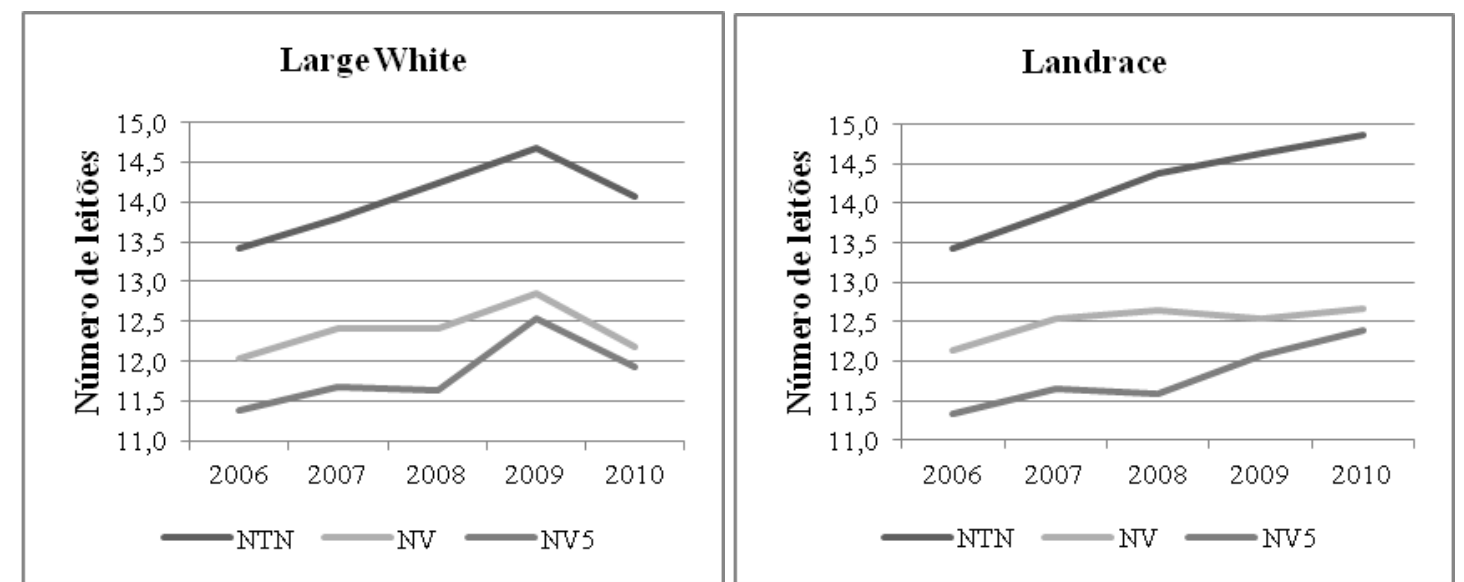

Figura 1. Evolução do NTN, NV e LV5 nas raças Landrace e Large White entre 2006 e 2010.

Tabela 2. Componentes de variância obtidos nas análises unicaracterísticas para número de leitões nascidos total (NTN), nascidos vivos (NV) e leitões vivos ao quinto dia (LV5) nas raças Large White e Landrace

\begin{tabular}{|c|c|c|c|c|c|c|c|c|c|c|}
\hline \multirow{3}{*}{ Modelo 1} & \multicolumn{5}{|c|}{ Large White } & \multicolumn{5}{|c|}{ Landrace } \\
\hline & \multicolumn{5}{|c|}{ Variância (leitões ${ }^{2}$ ) } & \multicolumn{5}{|c|}{ Variância (leitões ${ }^{2}$ ) } \\
\hline & $\sigma_{\mathrm{e}}^{2}$ & $\sigma_{a}^{2}$ & $\sigma_{m}^{2}$ & $\sigma_{p}^{2}$ & $\sigma_{f}^{2}$ & $\sigma_{e}^{2}$ & $\sigma_{a}^{2}$ & $\sigma_{m}^{2}$ & $\sigma_{p}^{2}$ & $\sigma_{f}^{2}$ \\
\hline NTN & 11,74 & 2,14 & - & - & 13,88 & 9,62 & 1,27 & - & - & 10,9 \\
\hline NV & 9,25 & 1,52 & - & - & 10,77 & 8,21 & 1,14 & - & - & 9,35 \\
\hline LV5 & 9,98 & 1,32 & - & - & 11,25 & 9,39 & 0,86 & - & - & 10,27 \\
\hline \multirow[t]{2}{*}{ Modelo 2} & \multicolumn{6}{|c|}{ Variância (leitões ${ }^{2}$ ) } & \multicolumn{4}{|c|}{ Variância (leitões ${ }^{2}$ ) } \\
\hline & $\sigma_{e}^{2}$ & $\sigma_{\mathrm{a}}^{2}$ & $\sigma_{m}^{2}$ & $\sigma_{p}^{2}$ & $\sigma_{f}^{2}$ & $\sigma_{e}^{2}$ & $\sigma_{a}^{2}$ & $\sigma_{m}^{2}$ & $\sigma_{p}^{2}$ & $\sigma_{f}^{2}$ \\
\hline NTN & 11,74 & 2,14 & 0,002 & - & 13,88 & 9,57 & 1,05 & 0,298 & - & 10,92 \\
\hline NV & 9,25 & 1,51 & 0,015 & - & 10,78 & 8,16 & 0,69 & 0,523 & - & 9,38 \\
\hline LV5 & 9,92 & 1,27 & 0,062 & - & 11,26 & 9,33 & 0,51 & 0,444 & - & 10,3 \\
\hline \multirow[t]{2}{*}{ Modelo 3} & \multicolumn{6}{|c|}{ Variância (leitões ${ }^{2}$ ) } & \multicolumn{4}{|c|}{ Variância (leitões ${ }^{2}$ ) } \\
\hline & $\sigma_{\mathrm{e}}^{2}$ & $\sigma_{\mathrm{a}}^{2}$ & $\sigma_{m}^{2}$ & $\sigma_{p}^{2}$ & $\sigma_{\mathrm{f}}^{2}$ & $\sigma_{\mathrm{e}}^{2}$ & $\sigma_{\mathrm{a}}^{2}$ & $\sigma_{m}^{2}$ & $\sigma_{p}^{2}$ & $\sigma_{f}^{2}$ \\
\hline NTN & 11,72 & 2,07 & - & 0,081 & 13,87 & 9,38 & 0,94 & - & 0,536 & 10,87 \\
\hline NV & 9,25 & 1,52 & - & 0,008 & 10,77 & 7,91 & 0,69 & - & 0,691 & 9,3 \\
\hline LV5 & 9,92 & 1,32 & - & 0,011 & 11,25 & 9,1 & 0,5 & - & 0,629 & 10,24 \\
\hline \multirow[t]{2}{*}{ Modelo 4} & \multicolumn{5}{|c|}{ Variância (leitões ${ }^{2}$ ) } & \multicolumn{5}{|c|}{ Variância (leitões ${ }^{2}$ ) } \\
\hline & $\sigma_{e}^{2}$ & $\sigma_{a}^{2}$ & $\sigma_{m}^{2}$ & $\sigma_{p}^{2}$ & $\sigma_{f}^{2}$ & $\sigma_{e}^{2}$ & $\sigma_{\mathrm{a}}^{2}$ & $\sigma_{m}^{2}$ & $\sigma_{p}^{2}$ & $\sigma_{\mathrm{f}}^{2}$ \\
\hline NTN & 11,73 & 2,1 & 0,017 & - & 13,88 & 9,41 & 0,85 & 0,267 & - & 10,9 \\
\hline $\mathbf{N V}$ & 9,25 & 1,51 & 0,013 & - & 10,78 & 7,94 & 0,47 & 0,439 & - & 9,34 \\
\hline LV5 & 9,92 & 1,27 & 0,053 & - & 11,25 & 9,12 & 0,32 & 0,375 & - & 10,27 \\
\hline
\end{tabular}


Tabela 3. Herdabilidade no sentido restrito $\left(h^{2}\right)$, herdabilidade do efeito genético materno $\left(h^{2}\right.$ mater $)$ e repetibilidade $(\mathrm{R})$ obtidas nas análises unicaracterísticas para número de leitões nascidos total (NTN), nascidos vivos (NV) e leitões vivos ao quinto dia (LV5) nas raças Large White e Landrace, e resultados de AIC e BIC para os modelos propostos

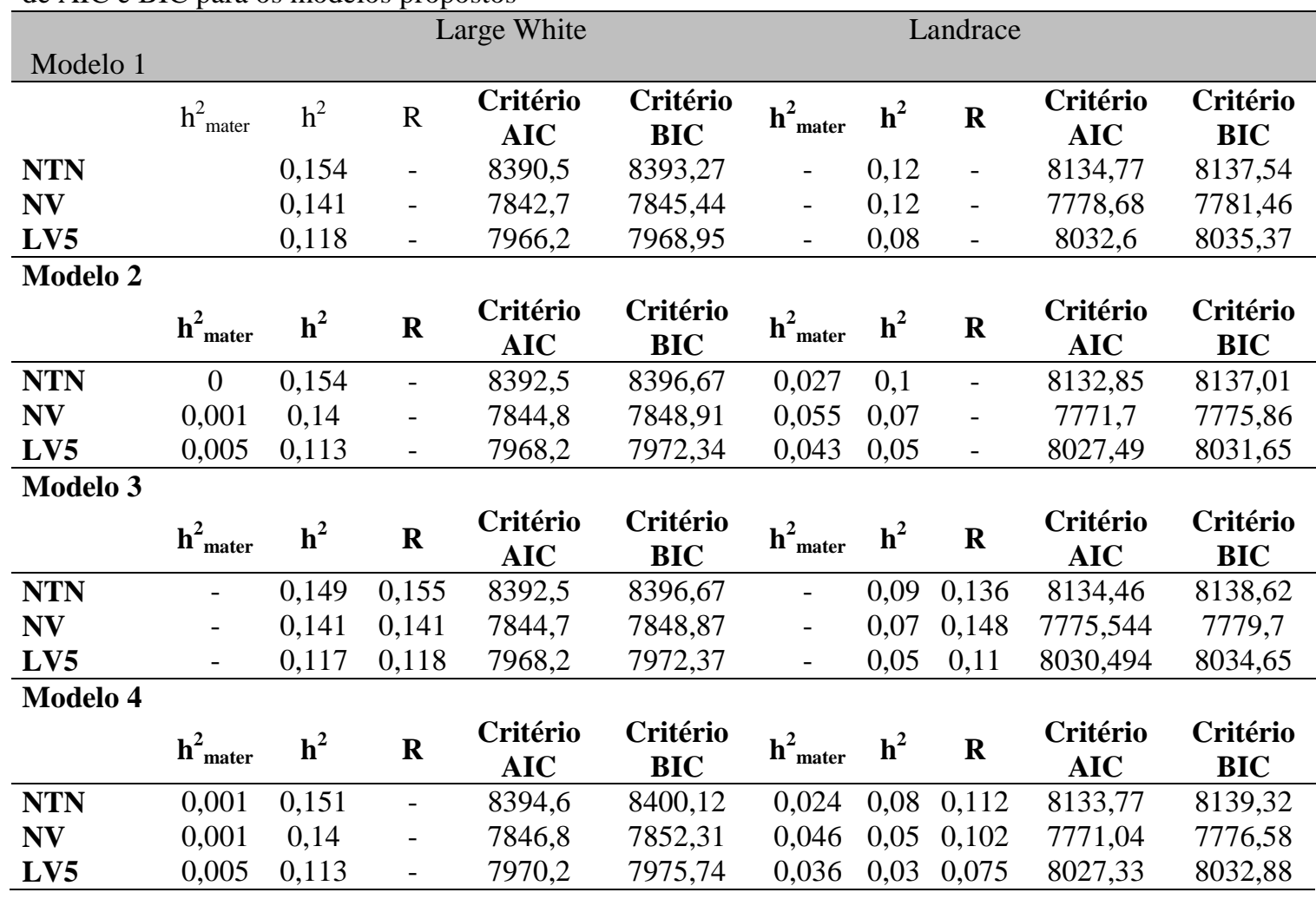

De acordo com os critérios AIC e BIC, para o estudo de características de leitegada, o modelo recomendado para LW foi o que continha apenas o efeito genético aditivo direto, pois as modificações nos dois critérios, com o acréscimo de parâmetros, não justificam a inclusão dos demais efeitos (genético materno e de ambiente permanente) para as características estudadas (NTN, NV e LV5). Já para a raça LD, o modelo que incluiu os efeitos maternos (modelo 2) teve o menor valor de BIC, o que justifica a incorporação desse efeito. Pelo critério AIC, o modelo que incluiu efeitos genéticos diretos, maternos e de ambiente permanente (modelo 4) foi o recomendado para a avaliação do LV5.

As estimativas de herdabilidade para NTN e NV foram maiores do que as estimadas para LV5 em ambas as raças, em acordo com as observações feitas por Su et al. (2007), que observaram que isso pode ser parcialmente explicado pelo efeito do longo tempo de seleção para NTN e NV comparativamente a LV5. Para o modelo 1, a estimativa de herdabilidade para NV foi de 0,14 para LW e 0,12 para LD. Irgang et al. (1994) relataram estimativas de herdabilidade de 0,09 para LW e 0,14 para LD para o primeiro parto, usando o modelo 1 . A estimativa de herdabilidade materna para NV na raça $\mathrm{LW}$ foi diferente dos valores relatados por Irgang et al. (1994), que observaram valores que variaram de zero a 0,13 , dependendo da ordem de parto da fêmea. Por outro lado, as estimativas dessa mesma quantidade no presente estudo não ultrapassaram 0,01. Para LD, a estimativa de herdabilidade materna obtida na análise aqui apresentada para NV foi de 0,06 para o modelo 2 e 0,05 para o modelo 4, situando-se dentro do estimado por aqueles autores, entre zero a 0,09, sendo menor nas primeiras ordens de parto, crescendo até o terceiro parto, e em seguida reduzindo. No presente estudo, a variabilidade estimada atribuída ao efeito genético materno for superior na raça $\mathrm{LD}$, em todos os modelos, em relação à raça $\mathrm{LW}$, o que pode justificar a inclusão desses efeitos para aquela raça. As 
estimativas de correlação entre os efeitos genéticos diretos e maternos foram positivos, apesar de terem sido muito próximos de zero na raça LW (resultados não apresentados). Esses valores contrariam resultados citados na literatura, que apontam correlações negativas e fortes entre os efeitos genéticos diretos e maternos (Irgang et al., 1994).

A variância de ambiente permanente foi maior na raça $L D$ e muito próxima de zero na raça $L W$ (Tabela 2). Pode-se perceber que, na raça LD, a inclusão dos efeitos genéticos maternos e de ambiente permanente resultou em diferentes partições da variabilidade total entre os efeitos aleatórios do modelo. Por outro lado, o mesmo não foi observado em $\mathrm{LW}$, na qual a variância genética aditiva foi maior e mais estável enquanto os outros efeitos aleatórios eram incluídos. Estes geralmente não conseguiram explicar proporções razoáveis da variância fenotípica. Como resultado, maiores estimativas de herdabilidade no sentido restrito foram observadas em LD para todas as características estudadas.

A partir de análises multicaracterísticas, considerando apenas o primeiro parto das fêmeas, para a raça $\mathrm{LD}$, as estimativas de correlações genéticas (e fenotípicas) entre NTN e NV; NTN e LV5; e NV e LV5 foram 0,98 $(0,83)$; $0,99(0,77)$ e $0,99(0,92)$. Para a raça $L W$, nessa mesma ordem, as estimativas foram $0,80(0,87)$, $0,85(0,83)$ e $0,99(0,95)$.

Alternativamente, a partir das correlações de Pearson entre os valores genéticos preditos para cada animal por meio das soluções para as análises univariadas, considerando todos os partos das fêmeas, as correlações genéticas obtidas entre NTN e NV; NTN e LV5; e NV e LV5 foram 0,$86 ; 0,68$ e 0,79 . Para a raça $L W$, nessa mesma ordem, as estimativas foram 0,90 , 0,84 e 0,97 .

Observou-se que as estimativas de correlação genética obtidas utilizando-se apenas os primeiros partos das fêmeas foram superiores às obtidas a partir dos valores genéticos. Isso pode indicar que variações não genéticas deixaram de ser consideradas com a exclusão de partos subsequentes ao primeiro. Nesse contexto, o número de dados foi reduzido a um terço do total, o que pode ter prejudicado as estimativas dos componentes de variância. Outro fator que pode ter influenciado fortemente as correlações é o fato de que as fêmeas primíparas normalmente apresentam NTN, NV e LV5 menores do que fêmeas multíparas. Quanto menor o número de leitões nascidos, maior tende a ser o peso individual dos leitões, o que colabora para a alta sobrevivência destes.

No rebanho estudado, o LV5 é uma característica que começou a ser objeto de seleção há relativamente pouco tempo, o que pode ser favorável para a estimação dos componentes de variância. Mais especificamente, o banco de dados possuía apenas cerca de três gerações de seleção para LV5, considerando-se um intervalo médio de geração para machos e fêmeas de 1,7 anos. Apesar desse aspecto, a tendência genética e fenotípica das duas raças para LV5 parecem ser consistentes (Fig. 2, para LD; Fig. 3, para LW). Isso pode estar ocorrendo em razão de alterações nas frequências alélicas nesse início de processo de seleção, o que justificaria também as flutuações que acontecem nas médias ao longo dos anos. A evolução é mais explícita em LW do que em LD, o que é consistente com os resultados apresentados na Tabela 2, em que LW apresentou maior variância fenotípica para LV5 (11,249 leitões ${ }^{2}$ para LW versus 10,267 leitões ${ }^{2}$ para LD) e especialmente maior variância genética aditiva (1,322 leitões ${ }^{2}$ para $\mathrm{LW}$ versus 0,868 leitões $^{2}$ para LD) para LV5. Portanto, de acordo com os resultados encontrados, a seleção para LV5 na raça LW tenderia a ser mais efetiva. Comparando-se as médias fenotípicas para LV5 nas raças $\mathrm{LD}$ e $\mathrm{LW}$, apresentadas na Tabela 1 , e com base nos componentes de variância estimados, pode-se inferir que a raça LW foi geneticamente superior à raça LD nessa característica, com possibilidade de resposta à seleção superior, em parte devido à sua maior variância genética aditiva e ao maior desvio padrão fenotípico. 


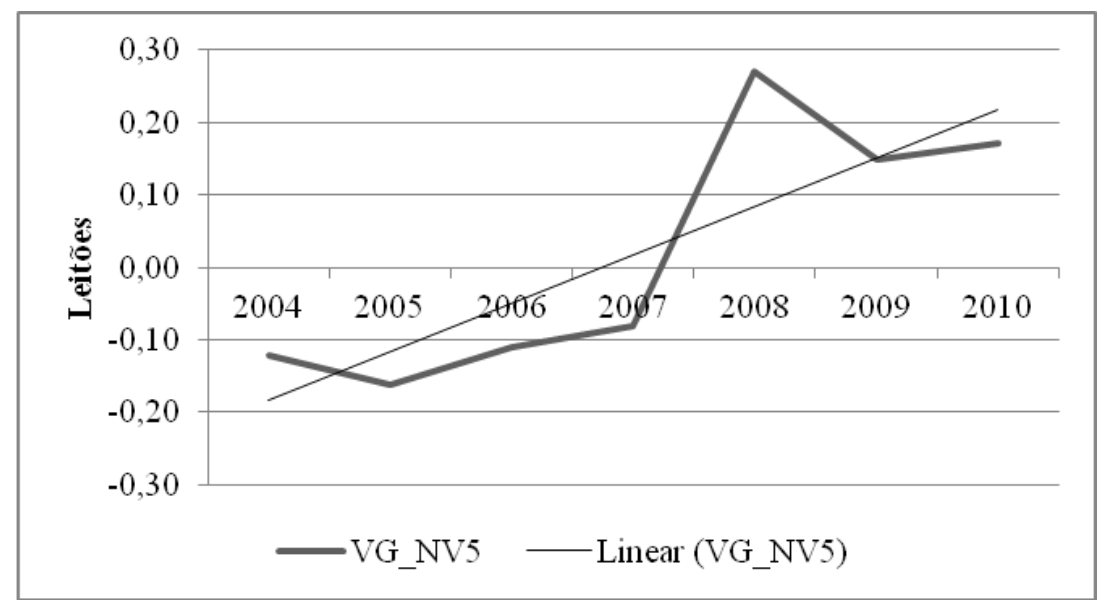

Figura 2. Evolução do valor genético das fêmeas, por ano de nascimento, para o LV5 na raça Landrace.

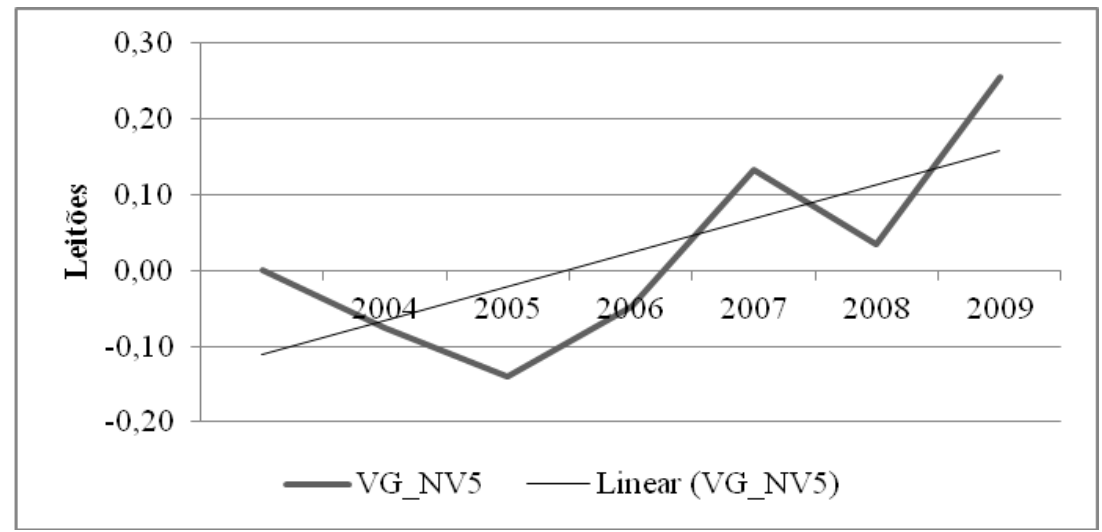

Figura 3. Evolução do valor genético das fêmeas, por ano de nascimento, para o LV5 na raça Large White.

Considerando-se uma taxa de reposição média de $70 \%$ para fêmeas, com intensidade de seleção de cerca de $7 \%(\mathrm{i}=1,90)$ e reposição de $120 \%$ para machos, o ganho genético esperado por geração para a característica LV5 seria de 0,59 leitões na LD e de 0,92 leitões na LW. Observando-se os quadros da evolução genética (Fig. 2 e Fig. 3), deduz-se que, em três gerações, houve um ganho genético de 0,33 leitões na LW e de 0,43 leitões na $\mathrm{LD}$, o que equivale a ganho de 0,11 leitões por geração LW e 0,14 leitões por geração $L D$, ou seja, cerca de um quinto do ganho genético esperado; e com uma inversão, pois a raça LD teve maior ganho genético no período do que a raça $\mathrm{LW}$, sendo que se esperaria o contrário em virtude da menor herdabilidade e menor variância fenotípica naquela raça.

\section{CONCLUSÕES}

O melhor modelo estatístico para estimar valores genéticos para LV5 incluiu apenas o efeito aditivo genético direto para LW, e os efeitos genéticos diretos, materno e de ambiente permanente para LD. A variabilidade genética observada foi superior à de outros estudos para a característica LV5, e a estimativa de herdabilidade foi de 0,08 para LD e de 0,12 para LW. A seleção para a característica LV5 tem se mostrado eficiente e se refletiu no aumento observado ao longo dos anos no número de leitões vivos ao quinto dia nas duas raças estudadas. Concluiu-se que a seleção para aumento do LV5, por ser uma variável de fácil mensuração no dia a dia dos programas de melhoramento genético de suínos, é promissora, pois se reflete no aumento da sobrevivência dos leitões e, consequentemente, no aumento do número de leitões desmamados por leitegada. 


\section{REFERÊNCIAS}

AKAIKE, $H$. Information Theory and an Extension of the Maximum Likelihood Principle. In: PETROX, B.N. e CASKI, F. (Ed.) Second International Symposium on Information Theory, Budapest: Akademiai Kiado, 1973, p.267.

BOUQUET, A.; CANARIO, L.; LIGONESCHE, B.; BIDANEL, J.P. Genetics parameters of litter size, piglet preweaning mortality and growth in French Landrace pigs. In: WORLD CONGRESS ON GENETICS APPLIED TO LIVESTOCK PRODUCTION, 8., 2006. Anais... Belo Horizonte, 2006.

FÁVERO, J.A.; FIGUEIREDO, E.A.P. Evolução do melhoramento genético de suínos no Brasil., Rev. Ceres, v.4, p.420-427, 2009.

IRGANG, R.; FÁVERO, J.A.; KENNEDY, B.W. Genetic parameters for Litter size of different parities in Duroc, Landrace and Large White sows. J. Anim. Sci., v.72, p.2237-2246, 1994.

LUND, M.S.; PUONTI, M.; RYDHMER, L.; JENSEN, J. Relationship between litter size and perinatal and pre-weaning survival in pigs. $J$. Anim. Sci., v.74, p.2117-2222, 2002.

MEYER, K. WOMBAT - A tool for mixed model analyses in quantitative genetics by restricted maximum likelihood (REML). $J$. Zhejiang Univ. Sci., v.8, p.815-821, 2007.

STATISTICAL Analysis Sistem - SAS INSTITUTE INC. SAS/STAT. User's Guide: stat. Release 8.1 Edition. Cary, 1292p. 2001.

SCHWARZ, G., Estimating the Dimension of a Model .The Annals of Statistics, v.6, p.461-464, 1978.

SU, G.; LUND, M.S.; SORENSEN, D. Selection for litter size at day five to improve litter size at weaning and piglet survival rate. J. Anim. Sci., v.85, p.1385-1392, 2007.

SU, G.; SORENSEN, D.; LUND, M.S. Analysis of variance components for piglet survival using threshold model. In: WORLD CONGRESS ON GENETICS APPLIED TO LIVESTOCK PRODUCTION, 8., 2006. Anais... Belo Horizonte, 2006. 